\title{
Trastorno del espectro autista: Diagnóstico clínico y test ADOS
}

\section{Autism spectrum disorder: Clinical diagnosis and ADOS Test}

\author{
María Cecilia González ${ }^{\mathrm{a}}$, Macarena Vásquez ${ }^{\mathrm{b}, \mathrm{c}}$, Marta Hernández-Chávez ${ }^{\mathrm{a}}$ \\ aUnidad de Neurología Pediátrica, División de Pediatría, Pontificia Universidad Católica de Chile \\ bDepartamento de Neurología, Facultad de Medicina, Pontificia Universidad Católica de Chile \\ 'Psicóloga
}

Recibido el 8 de agosto de 2018; aceptado el 25 de octubre de 2018
Palabras clave:

Autismo;

trastorno del espectro

autista;

test ADOS;

$\mathrm{K}-\mathrm{BIT}$;

WISC IV;

Escala de Vineland

\section{Keywords:}

Autism; Autism

Spectrum Disorders;

ADOS test;

K-BIT; WISC IV;

Vineland Scale

Correspondencia:

Dra. Marta Hernández

mhernand@med.puc.cl 
of a series of 50 consecutive cases, which were clinically diagnosed with ASD between 2012 and 2016. Children aged between 3 to 10 years at the time of the ADOS-G test and language of at least one word were included. The children were evaluated neuropsychologically (functionality, intellectuality and ADOS test). We compared the median age to the neurological diagnosis, according to the autistic symptomatology and cognitive level. Results: The ADOS test corroborated an ASD in 44 children (88\%), 93.1\% were males. The average age at clinical diagnosis and ADOS test was $48.2 \pm 19.3$ and $62.6 \pm 23.3$ months. The neurological consultation in $72 \%$ of cases was parental/educator initiative due to symptoms such as social interaction disorder and language delay. The autistic symptomatology was mild, moderate and severe in $34.1,47.7$ and $18.2 \%$ respectively. In five of 27 children who were neuropsychologically evaluated cognitive deficits were detected. The median age at diagnosis was significantly lower in children with severe autism symptoms vs the ones with mild-moderate symptoms (p-value 0.024 ). Conclusion: Autistic symptoms determine the early consultation; therefore, it is necessary to guide the general and educational population as well as health professionals regarding these symptoms.

\section{Introducción}

Los criterios diagnósticos del trastorno del espectro autista (TEA) han cambiado en los últimos años, respondiendo al desafío que supone la alta variabilidad clínica con la que se presenta esta condición. El DSM-V (Manual de Diagnóstico y Estadístico de los Desórdenes Mentales) define el TEA como un desorden persistente y heterogéneo del neurodesarrollo, y categoriza los síntomas en dos grupos: a) deficiencias en la comunicación e interacción social y b) patrones de comportamiento restrictivo y repetitivo. Se incluye en este espectro al Síndrome de Asperger, al trastorno desintegrativo infantil y al trastorno generalizado del desarrollo no especificado ${ }^{1}$.

En el 2012, de acuerdo a lo reportado por 11 sitios de vigilancia de TEA en USA, la prevalencia fue de 14,6 por 1.000 en niños de 8 años ( 1 en 68), con una razón de 4,5:1 para sexo masculino ${ }^{2}$. La prevalencia ha ido en aumento en las últimas décadas, posicionándose como la segunda discapacidad del desarrollo más frecuente, después de la discapacidad intelectual ${ }^{3,4}$.

Una de las herramientas más ampliamente utilizadas para el diagnóstico de TEA es el test ADOS (Autism Diagnostic Observation Schedule), iniciado en los años $80^{35,6}$. El ADOS-G (genérico) es una evaluación estandarizada, semiestructurada de la interacción social, comunicación, juego imaginativo y uso de materiales para niños, jóvenes y adultos que pudieran tener un TEA. Tiene 4 módulos, cada uno de ellos con algoritmos diagnósticos y que permite al examinador observar el comportamiento a distintos niveles de desarrollo y lenguaje ${ }^{7}$. Este instrumento es sensible y específico y la última versión disponible en español corresponde al ADOS-2, que incluye mejoras y novedades como el diseño de un módulo para niños pequeños (12-30 meses) que se denomina Módulo T, así como la revisión de los algoritmos de los módulos 1-38.
Dado el continuo desarrollo en los criterios específicos del TEA, su relación con la prevalencia y otras características clínicas (sesgo en relación al sexo, edad de diagnóstico, etc.) se hace relevante una descripción detallada de la heterogeneidad del fenotipo autista en nuestra población.

Nuestro estudio tiene como objetivo describir el perfil clínico en una cohorte de niños con trastorno del espectro autista, desde su derivación a especialista hasta la realización del test ADOS-G que apoyará dicho diagnóstico. Se describirán los procesos, desde los primeros síntomas pesquisados por la madre, hasta la certificación diagnóstica.

\section{Pacientes y Método}

Este estudio fue realizado en la Unidad de Neurología Pediátrica de la PUC entre 2012-2016. Es un estudio descriptivo de una serie de casos consecutivos que se diagnostican clínicamente (examen neurológico y test ADOS-G). El diagnóstico clínico de TEA fue realizado por neurólogo pediatra según criterios de DSM-IV y posteriormente fueron referidos a evaluación neuropsicológica para evaluación de funcionalidad, intelectualidad y Test ADOS-G.

La historia clínica incluyendo sexo, antecedentes perinatales y familiares, primeros síntomas de TEA observados por la madre, edad al momento de la consulta con neurólogo pediátrico, motivo de la consulta, quién lo derivó a especialista, comorbilidades, escolaridad, estudios solicitados, antecedentes familiares, fueron obtenidos de las fichas clínicas. Todos los niños con sospecha de autismo fueron enviados a rehabilitación multidisciplinaria o talleres de habilidades sociales, se solicitaron exámenes durante sus controles posteriores. Se les extendió un informe escolar para integración o adecuaciones curriculares. El estudio fue aprobado por el Comité de ética institucional. 
Criterios de ingreso: Primera consulta por sospecha de TEA y ADOS-G compatible; edad entre 3 a 10 años al momento de la realización del Test ADOS-G; tener lenguaje hablado de al menos una palabra con significado. Criterios de exclusión: Vocalizaciones en la cual no se reconocen palabras ni aproximación a palabras; ser portador de una enfermedad genética cromosómica conocida o malformación severa o daño estructural del sistema nervioso central.

La evaluación neuropsicológica consistió en medición de la conducta adaptativa y cognitiva con el test de Vineland (Vineland Adaptative Behavior Scales Survey Form: 1-100), Kaufman Brief Intelligence (K-BIT), que provee Coeficiente intelectual $(\mathrm{CI})(\mathrm{M}=100$, $\mathrm{SD}=15$ ), para CI total, verbal y no verbal y Wechsler Intelligence Scale for Children-Fourth Edition (WISCIV), aplicable a niños y adolescentes de edades comprendidas entre 6 años 0 meses y 16 años 11 meses, que también arrojan CI de los mismos tipos descritos anteriormente ${ }^{10}$.

El test ADOS-G, evaluó si los pacientes tenían o no sintomatología del espectro autista, y se categorizó en carga sintomática leve, moderada o severa.

La evaluación neuropsicológica fue realizado por neuropsicóloga con certificación en evaluación Test ADOS.

\section{Análisis estadístico}

Las variables demográficas categóricas se calcularon en número y porcentaje y para las variables numéricas se calcularon promedios y desviación estándar (DE) o medianas y rango intercuartil (RIC) según distribuían normal o no. La comparación de medianas de edad y RIC al inicio de síntomas (reportados por la madre), al momento de la consulta neurológica y test ADOS$G$ vs gravedad de sintomatología autista se realizó con la prueba U de Mann-Whitney dicotomizado en levemoderado vs grave $(\mathrm{p}<0,05)$.

\section{Resultados}

De los 50 niños testeados, 44 cumplieron criterios de inclusión por tener ADOS-G concordante. Estos 44 casos se describen según protocolo (figura 1).

El 93,2\% (41/44) fueron niños, con una razón niño/niña de 13,6/1. El promedio de edad a la evaluación neurológica y aplicación del test ADOS-G fue 48,2 $( \pm 18,3)$ y $62,6( \pm 23,3)$ meses. En $4 / 44$ niños hubo antecedentes familiares de TEA y 13/44 tuvo morbilidad perinatal (tabla 1).

Las principales solicitudes para una primera evaluación neurológica en los 44 niños ocurrieron por consulta espontánea de los padres en $18 / 44$ y a solicitud del establecimiento educacional en 16/44. Otras solicitudes fueron derivación de otro médico (7/44 niños) y por otro profesional de la salud (psicólogo y fonoaudiólogo) en 3/44.

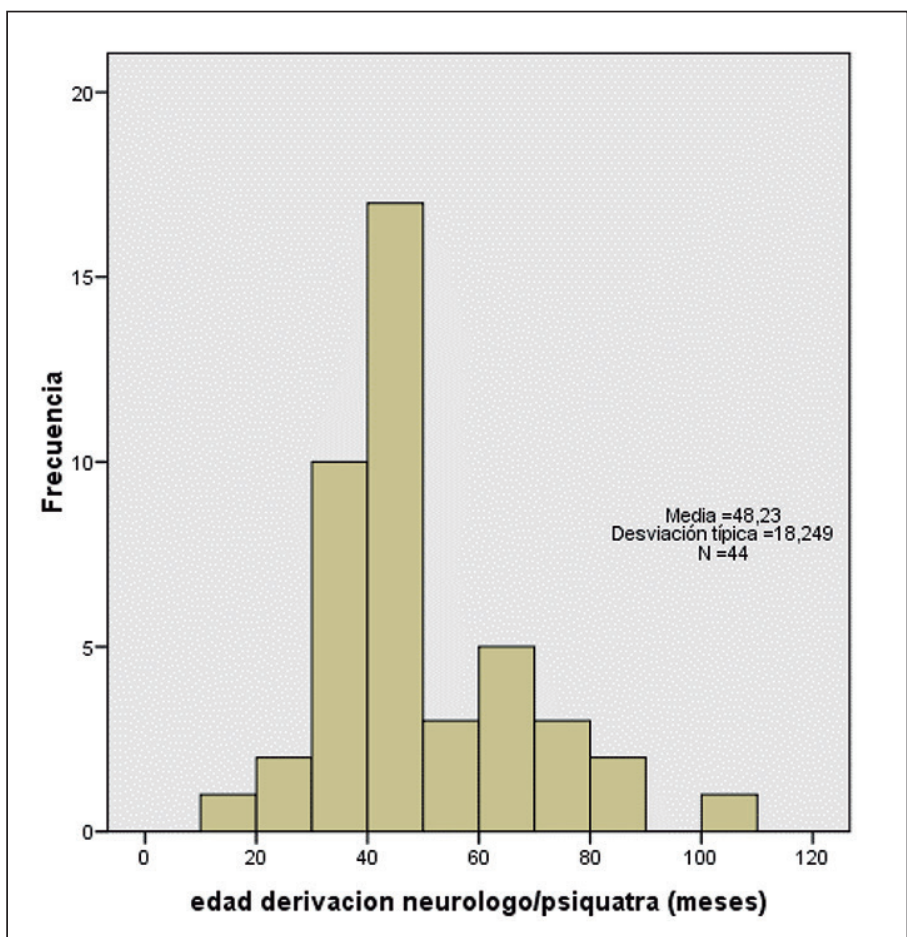

Figura 1. Edad de derivación al neurólogo/psiquiatra en 44 niños con trastorno del espectro autista.

Tabla 1. Características de 44 niños con TEA evaluados entre el año 2012-2016, PUC, Santiago, Chile

\begin{tabular}{lc}
\hline Características & $\mathrm{n}(\%)$ \\
\hline Sexo masculino & $41(93,2)$ \\
Edad $1^{\circ}$ consulta por TEA (m) promedio, DE & $48,2( \pm 19,3)$ \\
Antecedentes de familiar con TEA & $4(9,1)$ \\
Antecedentes perinatales (42/44) & $13(31)$ \\
Prematurez & $3(7,1)$ \\
Hiperbilirrubinemia & $3(7,1)$ \\
Sufrimiento fetal agudo & $2(4,8)$ \\
Macrosomía & $2(4,8)$ \\
Otro (SHE, DPNI, TT) & $3(7,1)$ \\
Comorbilidades & \\
Dificultades en el Aprendizaje & $27(61,4)$ \\
Trastorno del sueño & $14(31,8)$ \\
Trastorno alimentario & $12(27,3)$ \\
Déficit Intelectual & $6(23,1)$ \\
Retraso del desarrollo motor & $8(18,2)$ \\
Epilepsia & $3(6,8)$ \\
\hline
\end{tabular}

SHE: síndrome hipertensivo del embarazo, DPNI: desprendimiento de placenta normoinserta, $\Pi$ : taquipnea transitoria. 
Tabla 2. Comparación de medianas de edad a la detección síntomas, diagnóstico clínico neurológica y Test ADOS y nivel funcional e intelectual vs carga de sintomatología autista, en niños con TEA, años 2012-2016, Universidad Católica, Santiago, Chile

\begin{tabular}{lccc}
\hline & & Sintomatología según ADOS \\
\cline { 2 - 3 } Edad (meses) & Severa (8/44) & Leve-Moderada (36/44) & valor $p$ \\
\hline Detección síntomas según la madre, mediana, RIC & $18.2(20)$ & $24.1(16.5)$ & 0,15 \\
Diagnóstico por neurólogo, mediana, RIC & $36(14,7)$ & $44,5(24.7)$ & 0,024 \\
Edad al Test ADOS, mediana, RIC & $51.5(20)$ & $60.3(33.5)$ & 0,06 \\
\hline & & Nivel Intelectual y funcional \\
\hline Derivación a neurólogo, mediana, RIC & $\leq 70(n=10)$ & $60.1(30)$ & 0,21 \\
\hline RIC: Rango intercuartil. TEA:trastorno del espectro autista. & $56.5(35.3)$ &
\end{tabular}

En la anamnesis realizada a los 44 casos, las madres refirieron haber percibido alguna alteración del neurodesarrollo (primer síntoma), a una edad promedio de 26,23 meses (DS 14,0). De dicha sintomatología las alteraciones del lenguaje y alteraciones en la interacción social correspondieron a 26/40 y 17/40. En 6/40 casos se describió un cuadro regresivo con pérdida de lenguaje e interacción social. En los casos restantes (4 casos) relataron que "siempre" fueron distintos, sin definir el síntoma principal.

Entre los exámenes solicitados y realizados, la evaluación de la función auditiva ocurrió en 17/44 (con potenciales evocados auditivos, audiometría e impedanciometría normales en $16 / 17$, solo 1 caso con hipoacusia de conducción leve). En 15/44 (34,1\%) se solicitó EEG estándar, de los cuales 3 de 15 realizados fueron anormales, 2 con actividad epileptiforme y 1 con una lentitud focal. Otros exámenes solicitados fueron neuroimágenes, estudios genéticos (cariotipo, array CGH, estudio molecular X frágil) y estudios metabólicos, pero en su mayoría no fueron realizados.

La evaluación neuropsicológica se realizó en 37/44 niños. En 10 se realizó evaluación de la conducta adaptativa (Vineland) y en 27 evaluaciones cognitivas (19 con K-BIT y 8 con WISC-IV). En el Vineland se obtuvo una mediana de 52 puntos (rango de 20-97). En los 27 niños en que se realizó una evaluación cognitiva, se obtuvo una mediana en escala verbal de 85 (58-126), ejecutiva de 82 (52-119) y total de 83 (44-126). En 5 niños se detectó un CI menor de 70 (déficit cognitivo).

El test ADOS-G definió una carga de sintomatología autista leve a moderada en 36 niños $(81,8 \%)$ v/s $8(18.2 \%)$ con sintomatología autista grave y hubo diferencias significativas entre las medianas de edad en ambos grupos a la primera consulta neurológica (p 0,024) (Tabla 2).

Otras comorbilidades detectadas, aparte del déficit cognitivo, fueron trastorno de aprendizaje en
$27 / 44(61,4 \%)$, trastornos del sueño en $14 / 44(31,8 \%)$, trastorno alimentario en $12 / 44(27,3 \%)$, retraso del desarrollo motor en $8 / 44(18,2 \%)$ y epilepsia en $3 / 44$ $(6,8 \%)$.

En 27/44 casos, los niños asistían a jardín infantil o colegio con integración y 10 de ellos iban además a escuela de lenguaje. En 13/44 casos, solo asistían a escuela de lenguaje o colegio especial ( 10 y 3 niños respectivamente) y 4/44 no estaban escolarizados.

En relación a la terapia farmacológica, 12/44 recibía melatonina, risperidona, metilfenidato o sertralina, sola o combinada y antiepilépticos. Tres niños asistían a terapia alternativa de hipoterapia

Los 6 niños cuyo ADOS no reunieron criterios de TEA, no fueron analizados en esta muestra, pero tuvieron seguimiento de al menos de 1 año y sus diagnósticos fueron mutismo selectivo y fobia social (2/6), discapacidad intelectual (2/6) y disarmonía cognitiva y fobia social $(2 / 6)$.

\section{Discusión}

El impacto familiar y social del diagnóstico de TEA, asociado a la heterogeneidad de sus síntomas y a la falta de marcadores biológicos, impone una evaluación multidisciplinaria que nos permita una alta certeza diagnóstica. Un error diagnóstico asume costos emocionales y sociales evitables. Según algunos autores $^{5,9}$, el porcentaje de un diagnóstico erróneo con un examen clínico es de un 10-12\%. En nuestro trabajo, tuvimos 6/50 (12\%) niños cuyo Test ADOS excluyó un TEA a pesar de tener algunas conductas similares y cuyo seguimiento a largo plazo fue compatible.

Nuestra cohorte excluyó niños portadores de una enfermedad cromosómica, malformación o daño cerebral severo conocido e incluyó niños con lenguaje (al menos una palabra o gesto con significado), por ello 
creemos que se seleccionó un subgrupo de TEA con mayores habilidades cognitivas, definidos como TEA de alto funcionamiento (TEA-AF $)^{10,11}$. A pesar que el criterio de ingreso fue entre 36 meses a 10 años, creemos que la edad al diagnóstico de TEA-AF es mayor a la población general de TEA reportada por Nassar y Daniels $^{12,13}$ y mayor a los 18-24 meses recomendados para el tamizaje de ${\mathrm{M}-\mathrm{CHAT}^{14}}^{14}$. Esto hace necesario valorar los síntomas aportados por el medio que rodea al niño (padres y educadores) que es altamente sensible a las alteraciones del desarrollo.

El sorprendente predominio masculino encontrado en nuestros niños (13,6/1 vs 4/1 o 7/1 para TEA típico y TEA-AF) nos lleva a hipotetizar que tenemos un subdiagnóstico de TEA-AF en niñas ${ }^{15,16}$. Existe una creciente evidencia de un efecto de camuflaje entre las niñas con TEA, en particular entre las que no tienen discapacidad intelectual, lo que puede afectar el rendimiento en las medidas de diagnóstico estándar. Una de las hipótesis de Head et al. ${ }^{17}$ es que las niñas con TEAAF retienen las mayores habilidades sociales y emocionales que caracterizan a la población femenina porque utilizan habilidades cognitivas para responder a situaciones sociales ${ }^{18}$. Otra teoría es el "efecto protector femenino" ya que la mujer tendría un umbral genético más alto en relación a los hombres. También se han encontrado niveles de testosterona más altos en niñas con TEA que con desarrollo normal ${ }^{19}$.

La causa más frecuente de consulta fue la falta de interacción social (43\%), pilar fundamental del diagnóstico de TEA. La alteración del lenguaje o desarrollo atípico del lenguaje no está como criterio diagnóstico de TEA ${ }^{1}$ pero es descrito en el $86,4 \%$ de nuestros pacientes. Por otra parte, no se mencionaron como causa de consulta las conductas repetitivas y patrones restringidos del comportamiento, aun cuando se mencionaron a la anamnesis dirigida durante la consulta neurológica y la realización de Test ADOS. Este síntoma debe ser buscado en forma dirigida en toda consulta por alteración de lenguaje.

Con respecto a los tiempos en el proceso diagnóstico, 1 de cada 4 madres describió alteraciones del desarrollo antes del año de edad, pero la consulta o derivación a especialista ocurrió 20 meses más tarde. Esto concuerda con datos de Ozonoff y Martín ${ }^{20,21}$ que indican que el autismo puede ser diagnosticado en lactantes, basado en el informe parental y a pesar de ello la mediana de edad al diagnóstico es de 4 años ${ }^{22}$.

La discapacidad intelectual se describe en un $42 \%$ de la población con TEA ${ }^{16} \mathrm{y}$ en nuestra cohorte estuvo presente en el 5 de 27 niños evaluados con K-BIT y WISC (tabla 2). No hubo correlación significativa entre el nivel de CI y la mediana de edad al momento de la consulta neurológica, al revés de lo que ocurrió con la severidad de la sintomatología de TEA. Los niños con sintomatología autista grave consultaron antes que los niños con sintomatología leve-moderada.

El TEA puede representar la expresión final de varios factores etiológicos incluyendo condiciones genéticas con herencia conocida (ej. Esclerosis tuberosa), enfermedades metabólicas (ej. fenilcetonuria), infecciones congénitas (ej. rubeola congénita), anomalías estructurales (ej. hidrocefalia, agenesia cuerpo calloso) o anomalías neuroanatómicas y bioquímicas.

Para buscar estas etiologías es fundamental un examen clínico neurológico-psiquiátrico exhaustivo y evaluar el momento para realizar los exámenes neurofisiológicos, imageneológicos, genéticos y metabólicos adecuados. En la mayoría de los casos, estos son de gran costo y requieren sedación o anestesia general en el caso de estudios neurofisiológicos e imágenes cerebrales lo que limita aún más su realización. En nuestros casos se solicitaron una gran cantidad de exámenes que en su mayoría no se realizaron.

El estudio genético es uno de los más sugeridos en todas las cohortes de niños con autismo a nivel internacional, necesarios específicamente para consejo genético en caso de etiologías hereditarias ${ }^{23}$. En caso de regresión autista o sospecha de epilepsia, un EEG en sueño y vigilia deberá excluir un síndrome Landau-Kleffner o una encefalopatía de punta onda continua en sueño lento $u$ otro tipo de epilepsia que deteriora la comunicación, susceptible de mejorar con tratamiento antiepiléptico ${ }^{24}$. Las neuroimágenes o estudios metabólicos se solicitarán en caso de alta sospecha de lesión intracraneana o error congénito del metabolismo.

Las comorbilidades más frecuentes fueron dificultades en el aprendizaje, trastornos del sueño y alimentarios (61,4\% 31,8 y 27,3\%). Por ello los fármacos más indicados fueron metilfenidato y melatonina. Destaca poco uso de risperidona ${ }^{17}$.

A pesar que $40 / 44$ niños $(90,1 \%)$ estaba asistiendo a un establecimiento educacional, (jardín, colegio, escuela de lenguaje, escuela especial) cerca del 10\% (4/44) permanecían sin escolaridad. Ningún niño asistía a terapias de intervención educacional-conductual como ABA (applied behavior analysis) considerando los buenos resultados descritos por la literatura ${ }^{25}$ en programas iniciados precozmente e implementados en forma intensiva (más de $20 \mathrm{~h}$ por semana). Destaca la escasez de centros chilenos que realicen este tipo de terapias (comunicación personal del autor).

Entre las debilidades de nuestro trabajo están los criterios de ingreso que sesgaron la muestra hacia niños de mayor edad, con lenguaje y por tanto mejor nivel cognitivo. No se incluyeron niños menores porque el instrumento de evaluación de carga autista (ADOSG) e intelectualidad (K-BIT y WISC) es aplicable en mayores de tres años y niños con lenguaje. Creemos 
necesario, realizar nuevos estudios que incluyan una muestra de mayor número y menor edad (pre-escolares) para ser evaluados con los instrumentos actuales como ADOS-2, módulo T para niños de 12 a 36 meses y pruebas psicológicas como test Leiter-R (que permite evaluar intelectualidad en niños sin lenguaje).

\section{Conclusiones}

La carga de sintomatología autista, dado por alteraciones en interacción, comunicación e intereses restringidos es el síntoma que motiva una consulta temprana en padres y educadores. A pesar que existen criterios clínicos definidos en DSM IV y DSM V, factibles de detectar clínicamente, la alta variabilidad fenotípica del TEA relacionado con carga de sintomatología autista, capacidad cognitiva y lenguaje requiere un trabajo en equipo (familia, educadores, y equipo de salud) para una detección precoz ${ }^{26}$.

\section{Responsabilidades éticas}

Protección de personas y animales: Los autores declaran que los procedimientos seguidos se conformaron a las normas éticas del comité de experimentación humana responsable y de acuerdo con la Asociación Médica Mundial y la Declaración de Helsinki.

Confidencialidad de los datos: Los autores declaran que han seguido los protocolos de su centro de trabajo sobre la publicación de datos de pacientes.

Derecho a la privacidad y consentimiento informado: Los autores han obtenido el consentimiento informado de los pacientes y/o sujetos referidos en el artículo. Este documento obra en poder del autor de correspondencia.

\section{Conflicto de intereses}

Los autores declaran no tener conflicto de intereses.

\section{Referencias}

1. Grzadzinski R, Huerta M, Lord C. DSM-5 and autism spectrum disorders (ASDs): an opportunity for identifying ASD subtypes. Mol Autism. 2013;4(1):12-20.

2. Developmental Disabilities Monitoring Network Surveillance Year 2010 Principal Investigators, Centers for Disease Control and Prevention (CDC). Prevalence of autism spectrum disorder among children aged 8 years - autism and developmental disabilities monitoring network, 11 sites, US, 2010. MMWR Surveill Summ. 2014;63(2):1-21.

3. Lindsay WR, Carson D, O'Brien G, et al. A Comparison of Referrals With and Without Autism Spectrum Disorder to Forensic Intellectual Disability Services. Psychiatry, Psychology and Law. 2014,21(6):947-54

4. Yeargin-Allsopp M, Rice C, Karapurkar T, Doernberg N, Boyle C, Murphy C. Prevalence of autism in a US metropolitan area. JAMA. 2003;289(1):49-55.

5. Falkmer T, Anderson K, Falkmer M, Horlin C. Diagnostic procedures in autism spectrum disorders: a systematic literature review. Eur Child Adolesc Psychiatry. 2013, 22(6):329-40.

6. Gotham K, Risi S, Pickles A, Lord C. The Autism Diagnostic Observation Schedule: revised algorithms for improved diagnostic validity. J Autism Dev Disord. 2007;37(4):613-27.

7. Lord C, Risi S, Lambrecht L, et al. The autism diagnostic observation schedule-generic: a standard measure of social and communication deficits associated with the spectrum of autism. J Autism Dev Disord. 2000; 30(3):205-23.

8. Gotham K, Pickles A, Lord C. Trajectories of autism severity in children using standardized ADOS scores. Pediatrics. 2012;130(5):e1278-84.

9. Ousley O, Cermak T. Autism Spectrum Disorder: Defining Dimensions and Subgroups. Curr Dev Disord Rep. 2014;1(1):20-8

10. Lohr WD, Daniels K, Wiemken T, et al. The Screen for Child Anxiety-Related Emotional Disorders Is Sensitive but Not Specific in Identifying Anxiety in Children with High-Functioning Autism Spectrum Disorder: A Pilot Comparison to the Achenbach System of Empirically Based Assessment Scales. Front Psychiatry. 2017;8:138.

11. Berenguer C, Miranda A, Colomer C, Baixauli I, Rosello B. Contribution of Theory of Mind, Executive Functioning, and Pragmatics to Socialization Behaviors of Children with High-Functioning Autism. J Autism Dev Disord. 2018;48(2):430-41.

12. Daniels AM, Mandell DS. Explaining differences in age at autism spectrum disorder diagnosis: a critical review. Autism. 2014;18(5):583-97.

13. Nassar N, Dixon G, Bourke J, et al. Autism spectrum disorders in young children: effect of changes in diagnostic practices. Int J Epidemiol. 2009;38(5):1245-54.

14. Cuestionario M-CHAT Revisado de detección del Autismo en Niños
Pequeños con Entrevista de Seguimiento (M-CHART-R/F) [Internet]; 2016 [1]. Available from: http://mchatscreen.com/ wp-content/uploads/2017/01/M-CHATR_F_Espanol-Chile2017.pdf.

15. Ratto AB, Kenworthy L, Yerys BE, et al. What About the Girls? Sex-Based Differences in Autistic Traits and Adaptive Skills. J Autism Dev Disord. 2018;48(5):1698.

16. Baio J, Wiggins L, Christensen DL, et al. Prevalence of Autism Spectrum Disorder Among Children Aged 8 YearsAutism and Developmental Disabilities Monitoring Network, 11 Sites, United States, 2014. MMWR Surveill Summ. 2018;67(6):1-23.

17. Head AM, McGillivray JA, Stokes MA. Gender differences in emotionality and sociability in children with autism spectrum disorders. Mol Autism. 2014;5(1):19.

18. Bargiela S, Steward R, Mandy W. The Experiences of Late-diagnosed Women with Autism Spectrum Conditions: An Investigation of the Female Autism Phenotype. J Autism Dev Disord. 2016;46(10):3281-94.

19. Jacquemont $S$, Coe BP, Hersch M, et al. A higher mutational burden in females supports a "female protective model" in neurodevelopmental disorders. Am J Hum Genet. 2014;94(3):415-25.

20. Martínez M, Thomas KC, Williams CS, et al. Family Experiences with the Diagnosis of Autism Spectrum Disorder: System Barriers and Facilitators of Efficient Diagnosis. J Autism Dev Disord. 2018;48(7):2368-78. 
21. Ozonoff S, Heung K, Byrd R, Hansen $R$, Hertz-Picciotto I. The onset of autism: patterns of symptom emergence in the first years of life. Autism Res. 2008;1(6):320-8.

22. Christensen DL, Baio J, Van Naarden Braun K, et al. Prevalence and Characteristics of Autism Spectrum Disorder Among Children Aged 8 YearsAutism and Developmental Disabilities Monitoring Network, 11 Sites, United States, 2012. MMWR Surveill Summ.
2016;65(3):1-23.

23. Miller DT, Adam MP, Aradhya S, et al. Consensus statement: chromosomal microarray is a first-tier clinical diagnostic test for individuals with developmental disabilities or congenital anomalies. Am J Hum Genet. 2010;86(5):749-64.

24. Chen XQ, Zhang WN, Hu LY, Liu MJ, Zou LP. Syndrome of Electrical Status Epilepticus During Sleep: Epileptic Encephalopathy Related to Brain Development. Pediatr Neurol. 2016;56:35-
41.

25. Maglione M, Kadiyala S, Kress A, Hastings JL, O'Hanlon CE. TRICARE Applied Behavior Analysis (ABA) Benefit: Comparison with Medicaid and Commercial Benefits. Rand Health Q. 2017;6(2):10.

26. Randall M, Egberts KJ, Samtani A, et al. Diagnostic tests for autism spectrum disorder (ASD) in preschool children. Cochrane Database Syst Rev. 2018, 24;7:CD009044. 